VoL. 38 (1988) [41-54]

\title{
STABILITY AND J-DEPTH OF EXPANSIONS
}

\author{
JEAN-CAMILLE BIRGET
}

\begin{abstract}
In this paper $I$ prove that if a semigroup $S$ is stable then $\wedge_{L}(S)$ and $\wedge_{R}(S)$ (the Rhodes expansions), and $\wedge_{+}\left(S_{A}\right)$ (the iteration of those expansions) are also stable. I also prove that if $S$ is stable and has a J-depth function then these expansions also have a J-depth functon. More generally, if $X \rightarrow \rightarrow S$ is a $J^{*}$-surmorphism and if $S$ is stable and has a J-depth function then $X$ has a $J$-depth function. All these results are needed for the structure theory of semigroups which are stable and have a $\mathrm{J}$-depth function.

The techniques used were originally developed by the author to prove that $\wedge_{+}\left(S_{A}\right)$ is finite if $S$ is finite (later Rlodes found a much more direct proof of that result).
\end{abstract}

\section{INTRODUCTION}

\section{Stability and J-depth.}

Definition: A semigroup $S$ is $R$-stable if and only if no $J$-class of $S$ contains strict $R$-chains (equivalently, if $x \equiv_{J} y$ in $S$ and $x \geqslant_{R} y$ then $x \equiv_{R} y$ ). In a similar way one defines $L$-stable. A semigroup is stable if it is both $R$-and $L$-stable.

Definition: Let $s$ be a element of a semigroup $S$. The J-depth of $s$ is the length of the longest strictly ascending $J$-chain in $S$, starting with $s$. Equivalently, $\mathrm{J}$-depth $(\mathrm{s})=\max \left\{n \mid \exists s_{1}, \ldots, s_{n-1} \in S, s<_{J} s_{n-1} \cdots<_{J} s_{1}\right\}$. The J-depth of $s$ could be infinite.

A semigroup $S$ is said to have a J-depth function if and only if for every $s \in S$, the J-depth of $s$ is finite. (We will also say "the J-depth is defined in $S$ ").

For terms not defined in this paper see texts on algebraic semigroup theory, for example $([8,15,10])$.

An important propety of stable senugroups is that for them $J=D$ and Rees' theorem holds for every regular $D$-class.

\section{Received 15 September 1987}

This work was done in part at the Centre for Mathematical System Theory, University of Florida, Gainesville, FL 32611, and supported in part by U.S. Army Research Grant DAAG29-81-K-0136 and U.S. Air Force Grant AROSR81-0238 through the Centre for Math. System Theory. I would like to thank Professor R.E. Kalman for giving me the opportunity to work at the Centre for Mathematical System Theory, University of Florida, Gainesville FL 32611.

Copyright Clearance Centre, Inc. Serial-fee code: 0004-9729/88 \$A2.00+0.00. 


\section{Importance of the notions of stability and J-depth.}

Stability is a condition in Rees' theorem. The J-depth is needed for carrying out decompositions of a semigroup (for example to prove global theorems in the style of Kroln-Rhodes).

One can also view stability and existence of the $J$-depth function as a generalisation of finiteness: many theorems about finite semigroups carry over nicely in this case.

Stability is a generalisation of torsion (every torsion semigroup is stable). Torsion by itself is not a good enough generalisation of finite, being too much a local property.

Stability is a "local" property, in the sense that if refers only to each $J$-class separately. On the other hand, existence of the J-depth function is a purely global property of the $J$-order (which ignores the inside of the $J$-classes).

Semigroups that are stable and have a J-depth function arise for example as limits of finite semigroups (see [12]). This approach might be useful in the study of models of computation, especially parallel computation.

Structure theorem for semigroups that are stable and have a J-depth function.

Such semigroups have a structure theorem (generalising the case of finite semigroups) which combines Rees' theorem and the Krohn-Rhodes theorem. In the finite case that theorem was first stated and proved by Rhodes and Allen [14]. I proved a stronger version, which generalises to semigroups that are stable and have a J-depth function $[3,4]$. The results of the present paper are used in [4].

\section{Expansions.}

Simply speaking, an expansion associates with every semigroup $S$ a semigroup $E x(S)$ such that $S$ is a homomorphic image of $E x(S)$. A more precise definition can be found in $[6]$ or $[1]$, but will not be needed here.

The Rhodes expansion $\wedge_{L}(S)$ and $\wedge_{R}(S)$ of a semigroup $S$ are defined as follows (we will give the definition of $\wedge_{L}(S)$; that for $\wedge_{R}(S)$ is similar):

As a set $\wedge_{L}(S)$ consists of all strict $L$-chains of elements of $S$ (of the form $s_{n}<L$ $s_{n-1}<_{L} \cdots<_{L} s_{1}$, where $n>0$ and $\left.s_{n}, \ldots, s_{1} \in S\right)$. We define the multiplication in $\wedge_{L}(S)$ by:

$$
\begin{aligned}
\left(s_{n}<_{L} \cdots<_{L} s_{1}\right) \cdot\left(t_{k}<_{L} t_{k-1}\right. & \left.<L \cdots<_{L} t_{1}\right) \\
& =\operatorname{red}\left(s_{n} t_{k} \leqslant L \cdots \leqslant s_{1} t_{k} \leqslant L t_{k}<_{L} t_{k-1}<_{L} \cdots<_{L} t_{1}\right) .
\end{aligned}
$$

Here $\operatorname{red}(.$.$) is a reduction operation which transforms non-strict L$-chains into strict ones, according to the rules

$$
\operatorname{red}\left(\ldots \leqslant_{L} x<_{L} y \leqslant L \ldots\right)=\operatorname{red}\left(\ldots \leqslant_{L} x\right)<_{L} \operatorname{red}\left(y \leqslant_{L} \ldots\right) \text {, }
$$


and

$$
\operatorname{red}\left(\ldots \leqslant_{L} x \equiv_{L} y \leqslant_{L} z \leqslant_{L} \ldots\right)=\operatorname{red}\left(\ldots \leqslant_{L} x \leqslant_{L} z \leqslant_{L} \ldots\right) .
$$

In words: applying red to an $\leqslant L$-chain consists in reading the chain from right to left, and in keeping those elements that appear just before a strict $<_{L}$ symbol. We also assume that singleton chains are already reduced (that is for $s \in S: \operatorname{red}(s)=(s)$ ).

It is easy to check that with this multiplication $\wedge_{L}(S)$ is a semigroup. Moreover the map $\left(s_{n}<_{L} \ldots\right) \in \wedge_{L}(S) \mapsto s_{n} \in S$ is a homomorphism.

The Rhodes expansion $\wedge_{R}(S)$ is defined similarly (replacing $\leqslant_{L}$ by $\geqslant_{R}$; it is convenient to write $R$-chains in the descending direction $\left.s_{1}>_{R} \cdots>_{R} s_{n}\right)$.

For more information on the Rhodes expansion and its usefulness see [O, vol. B], $[17,11,1,6]$.

If $S$ is generated by a set $A \subseteq S$ then one can consider the subsemigroup $\wedge_{L}\left(S_{A}\right)$ of $\wedge_{L}(S)$ generated by the set of singleton $L$-chains $\{(a) \mid a \in A\}$. Usually $\wedge_{L}\left(S_{A}\right)$ is smaller that $\wedge_{L}(S)$, but in any case $S$ is a homomorphic image of $\wedge_{L}\left(S_{A}\right)$ (since $A$ generates all of $S)$. The semigroup $\wedge_{L}\left(S_{A}\right)$ is called "cutdown to generators". Both $\wedge_{L}(S)$ and $\wedge_{L}\left(S_{A}\right)$ were introduced by Rhodes.

One can apply $\wedge_{L}$ and $\wedge_{R}$ repeatedly to a semigroup, producing $\wedge_{R} \wedge_{L}(S)$, $\wedge_{L} \wedge_{L}(S), \wedge_{R} \wedge_{L} \wedge_{R}(S)$ etcetera. It is especially useful to keep always the same set of generators $A$ of $S$ and always to cut down to those. Then one has:

Theorem 1. (Tilson, see [1]). For every semigroup $S$ (generated by $A \subseteq S$ ):

$$
\wedge_{L} \wedge_{L}\left(S_{A}\right) \simeq \wedge_{L}\left(S_{A}\right)
$$

(where $\simeq$ denotes isomorphism) .

ThEorem 2. (Birget [1]). If $S$ is a finite semigroup (generated by $A \subseteq S$ ) then there exists $n$ such that

$$
\overbrace{\wedge_{R} \wedge_{L} \wedge_{R} \wedge_{L} \ldots}^{n+1 \text { times }}\left(S_{A}\right)=\overbrace{\wedge_{R} \wedge_{L} \wedge_{R} \wedge_{L} \cdots}^{n \text { times }}\left(S_{A}\right)
$$

that is applying more than $n$ expansions to $S$ (always cutting down to $A$ ) does not produce different semigroups.

The semigroup

$$
\overbrace{\wedge_{R} \wedge_{L} \wedge_{R} \wedge_{L} \ldots}^{n \text { times }}\left(S_{A}\right)
$$

(for that $n$ ) is denoted $\wedge_{+}\left(S_{A}\right)$.

Even if $S$ is infinite one can define $\wedge_{+}\left(S_{A}\right)$ as a projective limit of all the iterated expansions $\wedge_{L}, \wedge_{R} \ldots$ (cut down to a set $A$ of generators of $S$ ).

One has: 
TheOREM 3. (Birget [1]): $\wedge_{L} \wedge_{+}\left(S_{A}\right) \simeq \wedge_{+}\left(S_{A}\right) \simeq \wedge_{R} \wedge_{+}\left(S_{A}\right)$.

The expansions $\wedge_{L}\left(S_{A}\right), \wedge_{R}\left(S_{A}\right), \wedge_{+}\left(S_{A}\right)$ and their homomorphisins onto $S$ have interesting special properties. See $[1,11]$ for a more complete presentation; here we list only what we need.

Theorem 4. (Rhodes, see $[1,15]$ ). The map $\wedge_{L}\left(S_{A}\right) \rightarrow \rightarrow S$ (defined earlier) is an $R^{*}$-morphism.

By definition a surmorphism $h: S \rightarrow \rightarrow T$ is $R^{*}$ if and only if for every regular element $t$ of $T$ we have: $h^{-1}(t)$ is entirely included in one regular $R$-class of $S$. (This implies that the inverse image of a regular $R$-class of $T$ is equal to one regular $R$-class of $S$.)

Similarly, one can define $L^{*}$ and $J^{*}$ morphisms. The definition of $J^{*}$-morphisms is more complicated (since a $J$-class may contain regular and non-regular elements simultaneously). $\quad h: S \rightarrow \rightarrow T$ is $J^{*}$ if and only if for every regular element $t$ of $T, h^{-1}(t)$ consists of regular elements only and is entirely contained in one $J$-class. The map $\wedge_{R}\left(S_{A}\right) \rightarrow \rightarrow S$ is $L^{*}$. Every $L^{*}$ - (or $R^{*}{ }_{-}$) morphism is also $J^{*}$ and the composition of $J^{*}$-morphisms (respectively $L^{*}$ or $R^{*}$ ) is again $J^{*}$ (respectively $L^{*}$ or $R^{*}$ ). So the map $\wedge_{+}\left(S_{A}\right) \rightarrow \rightarrow S$ is $J^{*}$. See [1] and [15] for proofs and details. In this paper $J^{*}$-morphisms will be very important.

The main goal of this paper is to prove that: (1) If $S$ is stable then the expanded semigroups $\wedge_{L}\left(S_{A}\right), \wedge_{R}\left(S_{A}\right), \wedge_{+}\left(S_{A}\right)$ are also stable. (2) If $S$ is stable and has a J-depth function then $\wedge_{L}\left(S_{A}\right), \wedge_{R}\left(S_{A}\right), \wedge_{+}\left(S_{A}\right)$ also have J-depth functions. More generally, if $h: X \rightarrow \rightarrow S$ is a $J^{*}$-surmorphism and $S$ is stable and has a J-depth function, then $X$ also has a J-depth function.

Remark. Recently Rhodes [13] found a much simpler proof of Theorem 2 above (by observing that Brown's Lemma [7] can be applied to the morphism $\wedge_{+}\left(S_{A}\right) \rightarrow \rightarrow S$ when $S$ and $A$ are finite).

\section{STABILITY OF EXPANSIONS}

ThEOREM. If $S$ is stable then the expanded semigroups $\wedge_{L}\left(S_{A}\right), \wedge_{R}\left(S_{A}\right)$ and $\wedge_{+}\left(S_{A}\right)$ are also stable (where $A$ is a set of generators of $S$ ).

Moreover: If $S$ is a regular semigroup then $S$ is stable if and only if $\wedge_{L}\left(S_{A}\right)$ is stable (and the same is true with $\wedge_{L}\left(S_{A}\right)$ replaced by $\wedge_{R}\left(S_{A}\right)$ or $\wedge_{+}\left(S_{A}\right)$ ).

There exist (non-regular) semigroups $S$ for which $\wedge_{L}\left(S_{A}\right)$ or $\wedge_{R}\left(S_{A}\right)$ or $\wedge_{+}\left(S_{A}\right)$ is stable (and has a J-depth function), although $S$ itselt is not stable. (An example is given.)

Let us now prove the theorem. 
FACT 1. If $S$ is $L$-stable (that is $s \leqslant_{L} t$ and $s \equiv_{J} t$ implies $s \equiv_{L} t$ ) then $\wedge_{L}\left(S_{A}\right)$ is also $L$-stable.

Proof: Let $\underline{s}, \underline{t} \in \wedge_{L}\left(S_{A}\right)$ be such that $\underline{s} \equiv \equiv_{J} \underline{t}$ and $\underline{s} \leqslant_{L} \underline{t}$. Then $\underline{t}$ is of the form $\underline{t}=\left(t_{k}<_{L} t_{k-1}<_{L} \cdots<_{L} t_{1}\right)$. If we had $\underline{s}<_{L} \underline{t}$ then $\underline{s}$ would be of the form $\underline{s}=\left(s_{n}<_{L} \cdots<_{L} s_{k}<_{L} t_{k-1}<_{L} \cdots<_{L} t_{1}\right)$ with $s_{k} \equiv_{L} t_{k}$ (by definition of the $L$-order). Hence $s_{n}<_{L} t_{k}$.

Also, (by applying the morphism $\wedge_{L}\left(S_{A}\right) \rightarrow \rightarrow S$ to $\underline{s} \equiv J \underline{t}$ ): $s_{n} \equiv J t_{k}$. But if $S$ is $L$-stable we cannot have $s_{n}<_{L} t_{k}$ and $s_{n} \equiv_{J} t_{k}$.

In a similar way one proves that $\wedge_{R}\left(S_{A}\right)$ is $R$-stable.

The proof of the $R$-stability of $\wedge_{L}\left(S_{A}\right)$ (if $S$ is stable) is a little harder.

FACT 2. Let $X$ be any semigroup. Then: $X$ is $R$-stable if and only if for all $x, y \in X:\left(x \leqslant_{R} y\right.$ and $x \geqslant_{L} y$ implies $\left.x \equiv_{R} y\right)$.

That is in the definition of $R$-stability " $\equiv_{J}^{\prime \prime}$ can be replaced by " $\geqslant_{L}^{\prime}$.

Proof: Clearly, the left side of the "if and only if" implies the right side (since $x \leqslant_{R} y$ and $x \geqslant_{L} y$ implies $\left.x \equiv_{J} y\right)$.

Conversely, if $s \leqslant_{R} t$ and $s \equiv_{J} t$, then $t=a s b$ (for some $a, b \in S \cup\{1\}$ ), so $s \leqslant_{R} t \leqslant_{R} a s \leqslant_{L} s$. Hence $s \leqslant_{R} a s$, and $s \geqslant_{L}$ as. Therefore (since we assume the right side of the "if and only if") $s \equiv_{R}$ as. This implies (since $s \leqslant_{R} t \leqslant_{R}$ as): $s \equiv_{R} t$. So $X$ is $R$-stable.

FACT 3. If $S$ is $R$-stable then $\wedge_{L}\left(S_{A}\right)$ is $R$-stable.

Proof: By Fact 2 we only have to show that for all $\underline{s}, \underline{t} \in \wedge_{L}\left(S_{A}\right): \underline{s} \leqslant R \underline{t}$ and $\underline{s} \geqslant_{L} \underline{t}$ implies $\underline{s} \equiv_{R} \underline{t}$.

If $\underline{s} \leqslant_{R} \underline{t}$ we have $\underline{s}=\underline{t} . \underline{u}$ (for some $\underline{u} \in \wedge_{L}\left(S_{A}\right) \cup\{1\}$ ). Multiplying $\underline{s} \geqslant_{L} \underline{t}$ on the right by $\underline{u}$ yields $\underline{s} \cdot \underline{u} \geqslant_{L} \underline{t} \cdot \underline{u}(=\underline{s})$, therefore (by induction) we have:

For all $n \geqslant 0: \underline{s} \cdot \underline{u}^{n} \geqslant_{L} \underline{s}$.

Let $\underline{s}=\left(s<_{L} s_{i-1}<_{L} \cdots<_{L} s_{1}\right), \underline{u}=\left(u<_{L} u_{k-1}<_{L} \cdots<_{L} u_{1}\right)$. Then $\underline{s}^{n} \underline{u}^{n}$ $=\operatorname{red}\left(s . u^{n} \leqslant_{L} s_{i-1} u^{n} \leqslant_{L} \ldots \leqslant_{L} s_{1} u^{n} \leqslant_{L} u^{n} \ldots \leqslant_{L} u^{2} \leqslant_{L} u<_{L} u_{k-1}<_{L} \ldots<_{L} u_{1}\right)$ $\geqslant_{L} \quad\left(s<_{L} s_{i-1}<_{L} \cdots<_{L} s_{1}\right)$. This implies that the strict $L$-chain $\operatorname{red}\left(u^{n} \leqslant L u^{n-1} \leqslant_{L} \ldots \leqslant_{L} u^{2} \leqslant_{L} u\right)$ has length at most $|\underline{s}|$ (=length of the $L$-chain $\underline{s}$ ), which is a number depending only on $\underline{s}$ (not on $n$ ). Indeed a general property of the $L$-order of $\wedge_{L}\left(S_{A}\right)$ is that $\underline{x} \geqslant_{L} \underline{y}$ implies $|\underline{x}| \leqslant|\underline{y}|$.

It follows that in the $L$-chain $u \geqslant_{L} u^{2} \geqslant_{L} \ldots \geqslant_{L} u^{n-1} \geqslant_{L} u^{n} \geqslant_{L} \ldots$ the $\geqslant_{L^{-}}$ orders eventually all become $\equiv_{L}$. Precisely, there exists $m$ (depending only on $\underline{s}$ ) such that for all $n>0: u^{m+n} \equiv_{L} u^{m}$. Also, of course $u^{m} \geqslant_{R} u^{m+n}$. Thus (by $R$-stability of $S$ ): $u^{m} \equiv_{H} u^{m+n}$. So we have: 
There exists $m>0$ such that for all $n>0: u^{m+n}$ belongs to a fixed $H$-class of $S$.

The element $v=u^{m}$ satisfies $v \equiv_{H} v^{2}$. It follows that the $H$-class of $u^{m}=v$ is a group. Therefore, since the map $\wedge_{L}\left(S_{A}\right) \rightarrow \rightarrow S$ is an $R^{*}$-morphism (that is the inverse image of a regular $R$-class of $S$ is a regular $R$-class of $\wedge_{L}\left(S_{A}\right)$ ) we obtain:

There exists $m>0$ such that for all $n>0: \underline{u}^{m+n}$ belongs to a fixed $R$-class of $\wedge_{L}\left(S_{A}\right)$.

Let $\underline{v}=\underline{u}^{m}$. Then $\underline{v} \equiv_{R} \underline{v}^{2}$, so for some $\underline{y} \in \wedge_{L}\left(S_{A}\right) \cup\{1\}: \underline{v}=\underline{v}^{2} \cdot \underline{y}$. Also $\underline{s} \leqslant L \underline{s} \cdot \underline{u}^{m}(=\underline{s} \cdot \underline{v})$, by what was proved earlier.

Finally we can show that $\underline{t} \leqslant_{R} \underline{s}$. Since $\underline{t} \leqslant L \underline{s} \leqslant L \underline{s} \cdot \underline{v}$ we have: $\underline{t}=\underline{x} \cdot \underline{s} . \underline{v}$ (for some $\left.\underline{x} \in \wedge_{L}\left(S_{A}\right) \cup\{1\}\right)$. So $\underline{t}=\underline{x} \cdot \underline{s} \cdot \underline{v}^{2} \cdot \underline{y} \cdot\left(\right.$ since $\left.\underline{v}=\underline{v}^{2} \underline{y}\right)$, hence $\underline{t}=\underline{x} \cdot \underline{s} \cdot \underline{v} \cdot \underline{v} \cdot \underline{y}=\underline{t} \cdot \underline{v} \cdot \underline{y}$ (since $\underline{t}=\underline{x} \cdot \underline{s} \cdot \underline{v}$ ). Now (since $\underline{v}=\underline{u}^{m}=\underline{u} \cdot \underline{u}^{m-1}$ ) we obtain:

$\underline{t}=\underline{t} \cdot \underline{u}^{\prime} \underline{u}^{m-1} \underline{y}=\underline{s} \cdot \underline{u}^{m-1} \underline{y}$ (since $\underline{t} \cdot \underline{u}=\underline{s}$ ), which means: $\underline{t} \leqslant R \underline{s}$. (Remark: if $m=1$ we simply drop $\underline{u}^{m-1}$ ).

Similarly one proves that $\wedge_{R}\left(S_{A}\right)$ is $L$-stable if $S$ is $L$-stable.

From Facts 1 and 3 we obtain our theorem: if $S$ is stable then $\wedge_{L}\left(S_{A}\right)$ is stable (and similarly for $\wedge_{R}\left(S_{A}\right)$ ).

Let us finally prove that $\wedge_{+}\left(S_{A}\right)$ is stable if $S$ is stable. We will use the following two properties (see [1]):

(1) $\wedge_{+}\left(S_{A}\right)$ is a finitary projective limit (of the semigroups $\wedge_{L} \wedge_{R} \wedge_{L} \ldots\left(S_{A}\right)$ ).

(2) If $\leqslant$ denotes any Green relation $\left(\leqslant_{L}\right.$ or $\leqslant_{R}$ etcetra) then we have:

$\left(s_{n}\right)_{n \in N} \leqslant\left(t_{n}\right)_{n \in N}$ between elements in a finitary projective limit if and only if for all $n \in N: s_{n} \leqslant t_{n}$ in $S$.

Notice that for all $n: \overbrace{\wedge_{L} \wedge_{R} \wedge_{L} \ldots}^{n \text { limes }}\left(S_{A}\right)$ is stable if $S$ is stable (by applying our theorem for $\wedge_{L}$ and $\wedge_{R}$ inductively). Now the stability of $\wedge_{+}\left(S_{A}\right)$ (if $S$ is stable) follows from the following fact:

FACT 4. A finitary projective limit of stable semigroups is a stable semigroup.

Proof: (for $L$-stability, for example). If $\left(s_{n}\right)_{n \in N} \leqslant L\left(t_{n}\right)_{n \in N}$ and $\left(s_{n}\right)_{n \in N} \equiv J$ $\left(t_{n}\right)_{n \in N}$ in the projective limit then (by the property of the Green relations in finitary projective limits, stated above): $s_{n} \leqslant L t_{n}$ and $s_{n} \equiv_{J} t_{n}$ for all $n \in \mathrm{N}$. By the stability of $S$ this implies: $s_{n} \equiv L t_{n}$ for all $n \in N$. Thus (again by the property of the Green relations of finitary projective limits) we have $\left(s_{n}\right)_{n \in N} \equiv_{L}\left(t_{n}\right)_{n \in \mathbb{N}}$ in the projective limit.

Let us now deal with the case of regular semigroups, where the converse of our theorem is true: 
FACT 5. If $S$ is a regular semigroup and $\wedge_{L}\left(S_{A}\right)$ (or $\wedge_{R}\left(S_{A}\right)$ or $\wedge_{L}\left(S_{A}\right)$ ) is stable, then $S$ is stable.

Proof: (assuming stability of $\wedge_{+}\left(S_{A}\right)$, for example). Suppose $s, t \in S$ are such that $s \equiv{ }_{J} t$ and $s \leqslant_{L} t$. Then there exists $\underline{s}, \underline{t} \in \wedge_{+}\left(S_{A}\right)$ such that $s$ (respectively $t$ ) is the image of $\underline{s}$ (respectively $\underline{t}$ ) under $\wedge_{+}\left(S_{A}\right) \rightarrow \rightarrow S$, and $\underline{s} \leqslant_{L} \underline{t}$. Moreover since the map $\wedge_{+}\left(S_{A}\right) \rightarrow \rightarrow S$ is $J^{*}$ and $s$ and $t$ are regular we also have $\underline{s} \equiv J \underline{t}$. If $\wedge_{+}\left(S_{A}\right)$ is stable then this implies $\underline{s} \equiv_{L} \underline{t}$, hence (applying the morphism $\wedge_{+}\left(S_{A}\right) \rightarrow \rightarrow S$ ): $\underline{s} \equiv L \underline{t}$.

The same proof yields the slightly more general results:

Proposition. If $S$ is unstable with respect to regular elements (that is there exist regular elements $s, t \in S$ with $s \equiv_{J} t$, and $s<_{L} t$ or $\left.s<_{R} t\right)$ then $\wedge_{L}\left(S_{A}\right), \wedge_{R}\left(S_{A}\right)$ and $\wedge_{+}\left(S_{A}\right)$ are unstable.

For example, if $S$ contains the bicyclic semigroup then $\wedge_{L}\left(S_{A}\right), \wedge_{R}\left(S_{A}\right)$ and $\wedge_{+}\left(S_{A}\right)$ are unstable, and actually also contain the bicyclic semigroup, because the morphism $\wedge_{+}\left(S_{A}\right) \rightarrow \rightarrow S$ is in fact $D^{*}$. See [1].

Examples of semigroups $S$ which are unstable but for which $\wedge_{+}\left(S_{A}\right)$ is stable.

(By the above proposition these senugroups must be stable with respect to their regular elements.)

Examples are found among the idempotent-free semigroups, for which the following theorem holds:

ThEOREM. (Rhodes and Birget, $[11,1]$ ). $S$ is idempotent-free if and only if $\wedge_{L} \wedge_{R} \wedge_{L}\left(S_{A}\right)$ and $\wedge_{R} \wedge_{L} \wedge_{R}\left(S_{A}\right)$ are isomorphic to the free semigroup $A^{+}$.

The Baer-Levi senugroup (other examples can be found in [8, vol. 2]) is idempotentfree and unstable, but its $\wedge+$-expansion is the free semigroup, hence stable.

I suspect that if $S$ is stable with respect to its regular elements then $\wedge_{+}\left(S_{A}\right)$ will "usually" be stable. "Usually" means here that some additional natural condition on $S$ will suffice. For example I would guess that if $S$ is stable with respect to its regular elements and $S$ has the J-depth function then $\wedge_{+}\left(S_{A}\right)$ is stable (for a proof attempt use the null-regular layers, and the Falling Lemma, etcetera of the next two sections.) Notice that in $\wedge_{L} \wedge_{R}\left(S_{A}\right)$ and $\wedge_{R} \wedge_{L}\left(S_{A}\right)$ the Falling Lemma holds, no matter what $S$ is; this is proved in [1].

Since we will not need these results at this point we leave the question here.

\section{J-DEPTH FUNCTION OF EXPANSIONS}

In this section we will prove: 
MAIN Theorem. If $h: X \rightarrow \rightarrow S$ is a $J^{*}$-surmorphism, and if $S$ is stable and has a J-depth function, then $X$ has a $\mathrm{J}$-depth function.

Corollary. If $S$ is stable and has a J-depth function then the expansions $\wedge_{L}\left(S_{A}\right), \wedge_{R}\left(S_{A}\right)$ and $\wedge_{+}\left(S_{A}\right)$ also have J-depth functions.

We will give examples showing that if $S$ is not stable or does not have a $\mathrm{J}$-depth function then $\wedge_{L}\left(S_{A}\right)$ (or $\wedge_{R}\left(S_{A}\right)$ or $\wedge_{+}\left(S_{A}\right)$ ) does not necessarily have a J-depth function.

Recall that $h: X \rightarrow \rightarrow S$ is a $J^{*}$-morphism if and only if for every regular element $t \in S$ the set $h^{-1}(t)$ consists only of regular elements of $X$, and is entirely contained in one $J$-class of $X$.

In order to prove this theorem I will use a technique that I introduced in [1]; part of that technique will have to be generalised a little to work here. The technique consists of (1) the Falling Lemma, (2) the Null-Regular Layers of a semigroup.

\section{The Falling Lemma.}

The following property (introduced in [1]) is very useful and holds in many semigroups:

DEFINITION: A semigroup $S$ has the falling property if and only if for all $x, y \in S$ with $y \leqslant J x$ we have:

if $y$ and $x$ are both non-regular, and there is no regular element $J$-inbetween $y$ and $x$ (that is no regular element $r$ of $S$ satisfies $y \leqslant_{J} r \leqslant_{J} x$ ), then $x y<_{L} y$ and $y x<_{R} y$ (that is the products $x y$ and $y x$ fall below $y$ ).

In [1] I proved:

FACT 1. The following semigroups have the falling property:

(1) all finite semigroups;

(2) all semigroups that are finite- $J$-above (that is which satisfy: for all $s \in S$, the set $\left\{x \in S \mid x \geqslant_{J} s\right\}$ is finite);

(3) all torsion semigroups;

(Remark: (1) is of course a special case of (2) and of (3))

(4) all semigroups of the form $\wedge_{L} \wedge_{R}\left(S_{A}\right)$ or $\wedge_{R} \wedge_{L}\left(S_{A}\right)$, where $S$ is any semigroup (that is for any semigroup whatsoever, applying two Rhodes expansions $\wedge_{L} \wedge_{R}$ or $\wedge_{B} \wedge_{L}$ yields a semigroup which has the falling property).

Here we will generalise this result a little. The falling property is useful because it gives important information about the non-regular elements of a semigroup. 
DEFinition: Let $s$ be an element of $S$. The left-siabiliser of $s$, denoted $S_{\theta}$, is the subsemigroup $\{x \in S \mid x s=s\}$. The right-stabiliser of $s$ is $s=\{x \in S \mid s x=s\}$.

Definition: A semigroup $X$ is torsion modulo regular if and only if every element $x$ of $X$ has a power $x^{n}$ (for some $n>0$ ) which is regular.

Lemma 2. Suppose $S$ is such that the left-stabiliser and the right-stabiliser of every non-regular element is torsion modulo regular. Then $S$ has the falling property.

Proof: Suppose $y, x \in S$ with $y \leqslant J x$ and no regular element if $S$ is $J$-inbetween $y$ and $x$. Let us prove that $x y<_{L} y$ (the proof that $y x<_{R} y$ is similiar).

Assume on the contrary that $x y \equiv_{L} y$. So for some $u \in S \cup\{1\}: u x y=y$, hence $u x$ belongs to the left-stabiliser of $y$. Then, by assumption, some power $(u x)^{n}$ is regular. Now however $y=(u x)^{n} y \leqslant(u x)^{n} \leqslant x$ which means that the regular element $(u x)^{n}$ is $J$-inbetween $y$ and $x$.

From this lemma we derive a more interesting fact:

FACT 3. If $S$ is stable and has a J-depth function then $S$ has the falling property.

Proof: Let us show that the left-stabiliser of any non-regular element of $S$ is torsion modulo regular (the proof for right-stabilisers is similar).

Let $s$ be non-regular and suppose that $x s=s$. We must show that $x^{n}$ is regular, for some $n>0$. In $S$ we have the $J$-chain $x \geqslant_{J} x^{2} \geqslant_{J} \ldots \geqslant_{J} \cdot x^{k} \geqslant_{J} \ldots \geqslant_{J} x s=s$. Since $S$ has a J-depth function we have: there exists $m$ such that for all $n>0: x^{m} \equiv J$ $x^{m+n}$ (otherwise there would be no bound on the length of $J$-chains ascending from $s)$.

Next, by stability we have: there exists $m$ such that for all $n>0: x^{m} \equiv_{H} x^{m+n}$. Let $v=x^{m}$. Then $v \equiv_{H} v^{2}$. This inplies that the $H$-class of $x^{m}=v$ is regular (indeed if $v \equiv_{H} v^{2}$ then $v=a v^{2}=v^{2} b$ for some $a, b \in S \cup\{1\}$, hence $v^{2}=v \cdot v=v^{2} b a v^{2}$, so $v^{2}$ is regular).

Remark. Examples of semigroups that do not have the falling property are the BaerLevi semigroup (see [8, vol. 2] for a definition), or the extension of the semigroup $\left\{x, x^{2}=0\right\}$ by the natural integers.

\section{Null and Regular Layers of a semigroup.}

Let $S$ be a semigroup that is stable and has a J-depth function. $S$ can be partitioned into null (=non-regular) and regular layers as follows (see [ 1 ] for more details):

The first regular layer is

$\operatorname{Reg} 1=\left\{s \in S \mid s\right.$ is regular, and $x>_{J} s \Longrightarrow x$ is regular $\}$.

The first null layer is

Null1 $=\left\{s \in S-\operatorname{Reg} 1 \mid s\right.$ is null, and $\forall x \in S-\operatorname{Reg} 1: x>_{J} s \Longrightarrow x$ is null $\}$. 
More generally: Regk $=\left\{s \in S-\cup_{i<k}\right.$ Nulli $-\cup_{i<k} \operatorname{Reg} i \mid s$ is regular, and $\forall x \in$ $S-\cup_{i<k}$ Null $i-\cup_{i<k} \operatorname{Reg} i: x>_{J} s \Longrightarrow x$ is regular $\}$.

Null $k=\left\{s \in S-\cup_{i<k}\right.$ Null $i-\cup_{i \leqslant k} \operatorname{Reg} i \mid s$ is null, and $\forall x \in S-\cup_{i<k}$ Nulli$\cup_{i \leqslant k} \operatorname{Reg} i: x>_{J} s \Longrightarrow x$ is null $\}$.

It is clear that if $S$ is stable and has a J-depth function then every element of $S$ belongs to one and only one Null or Regular layer.

\section{Proof of the Main Theorem.}

Let us restate the theorem in a slightly stronger form.

Theorem. If $h: X \rightarrow \rightarrow S$ is a $J^{*}$-surmorphism and if $S$ is stable and has a $J$-depth function, then there exists an increasing function $f: N-\{0\} \longrightarrow N$ such that for all $x \in X$ :

$$
\mathrm{J}-\operatorname{depth}(x) \text { in } X \leqslant f(\mathrm{~J}-\operatorname{depth}(h(x)) \text { in } S) \text {. }
$$

(That is, the J-depth of $x$ is bounded by a function which depends only on $h(x)$.)

This theorem is a generalisation of Proposition 4.7 of $[1]$, which said: If $h: X \rightarrow \rightarrow$ $S$ is a $J^{*}$-surmorphism and if $S$ is finite, then $X$ has a $\mathrm{J}$-depth function; moreover the $\mathrm{J}$-depth function of $X$ is bounded by a number depending only on the maximal $\mathrm{J}$-depth in $S$.

The proof of the generalised theorem is similiar to the proof of the old Proposition 4.7 (of [1]), and goes by induction on the null-regular layers.

Although we yet have to prove that (under the conditions of the theorem) $X$ has a $J$-depth function it is clear from the definition of $J^{*}$-morphisms that the definitions of Null and Regular layers make sense for $X$, and that the $k$-th Null (respectively Regular) layer of $X$ equals $h^{-1}$ of the $k$-th Null (respectively Regular) layer of $S$. See also Section 2.7 of [1]. More precisely we have the following two facts:

FACT. If $h: X \rightarrow \rightarrow S$ is a $J^{*}$-surmorphism and if $S$ is stable then every $J$-class of $X$ consists either only of regular elements or only of non-regular elements.

Proof: Suppose $x, y \in X$ are such that $x \equiv_{J} y$ and suppose (by contradiction) that $x$ is regular and $y$ is non-regular. Since $h$ is a $J^{*}$-surmorphism $h(x)$ must be regular and $h(y)$ must be non-regular, but also $h(x) \equiv_{J} h(y)$ in $S$. This is however impossible in a stable semigroup.

FACT. If $h: X \rightarrow \rightarrow S$ is a $J^{*}$-surmorphism and if $S$ is stable and has a J-depth function, then $X$ has well-defined Null and Regular layers, that is, every element of $X$ belongs to one and only one Null or Regular layer. Moreover:

$$
\operatorname{Reg}_{X} k=h^{-1}\left(\operatorname{Reg}_{S} k\right), \quad \operatorname{Reg}_{S} k=h\left(\operatorname{Reg}_{X} k\right), \text { and }
$$




$$
\mathrm{Null}_{X} k=h^{-1}\left(\mathrm{Null}_{S} k\right), \quad \mathrm{Null}_{S} k=h\left(\mathrm{Null}_{X} k\right) .
$$

(where $\operatorname{Reg}_{X} k$ denotes the $k$-th regular layer of $X$, etc.)

Proof: The proof of Proposition 2.18 (of [1]) goes through without any changes.

Let us now go to the actual proof that $X$ has a J-depth function. The proof uses induction on null-regular layers (of $S$ or $X$ ).

For an element $x$ of Reg1 of $X$ we have $J$-depth $X(x)=J$-depth $(h(x))$, because $\operatorname{Reg} 1$ of $X$ and Reg1 of $S$ have isomorphic $J$-orders (since $h$ is $J^{*}$ ). So all elements of Reg1 of $X$ have a well defined J-depth, and J-depth ${ }_{X}(x)$ depends only on $\mathrm{J}_{\text {-depth }}(h(x))$. The function $f$ of the theorem is the identity function for $x \in \operatorname{Reg}_{X} 1$.

Assume now inductively that $J_{-}$depth $_{X}$ is defined on $\operatorname{Reg} 1 \cup \cdots \cup \operatorname{Null}(i-1) \cup \operatorname{Reg} i$ of $X$ and that in addition $\mathrm{J}_{-} \operatorname{depth}_{X}(x)$ is bounded by a function of $\mathrm{J}$-depth $\operatorname{det}_{S}(h(x))$ on these layers. More precisely, an increasing $f: N-\{0\} \rightarrow N$ has been found such that for all $x \in \operatorname{Reg} 1 \cup \cdots \cup N$ Null $(i-1) \cup \operatorname{Reg} i$ of $X: \mathrm{J}_{-} \operatorname{depth}_{X}(x) \leqslant f\left(\mathrm{~J}-\operatorname{depth}_{S}(h(x))\right)$. We must prove that this inductive hypothesis can be extended to Nulli.

Let $x \in$ Nulli of $X$ and consider a $<_{J}$-chain $x<_{J} x_{n-1}<_{J} \cdots<_{J} x_{0}<_{J} y_{P}<J$ $\cdots<_{J} y_{1}$, where $x_{n-1}, \ldots, x_{0} \in \operatorname{Null}_{X} i$ and $y_{P}, \ldots, y_{1} \in \operatorname{Reg} 1 \cup \ldots \cup \operatorname{Null}(i-1) \cup \operatorname{Reg} i$ of $X$. We will prove that the numbers $p$ and $n$ are bounded by a number depending only on $\mathrm{J}$-depth ${ }_{S}(h(x))$.

Proof for $p$ : since $y_{P} \notin N u l l i, p$ is bounded by $f\left(J_{-} \operatorname{depth}_{S}\left(h\left(y_{P}\right)\right)\right)$, by induction. Moreover, J-depth $\mathrm{J}_{S}\left(h\left(y_{P}\right)\right)<\mathrm{J}$-depth $\mathrm{de}_{S}(h(x))$, and $f$ is an increasing function, hence $p \leqslant f\left(J-\operatorname{depth}_{S}(h(x))\right)$. This holds, independently of the element $y_{P}$, as long as we keep $x$ fixed. Notice that we have not assumed that $x \in X$ has finite J-depth, but only that $h(x) \in S$ has finite J-depth.

Proof that $n$ (= length of any $\leqslant J$-chain in $X$ ascending from $x$, and entirely situated in Nulli of $X)$ is bounded by a function of $J$-depth ${ }_{S}(h(x))$ :

For the chain $x<_{J} x_{n-1}<_{J} \cdots<_{J} x_{j+1}<_{J} x_{j}<_{J} \cdots<_{J} x_{0}$ we may have $h\left(x_{j+1}\right)<_{J} h\left(x_{j}\right)$ in $S$ or $h\left(x_{j+1}\right) \equiv_{J} h\left(x_{j}\right)$ in $S$. Certainly the case " $h\left(x_{j+1}\right)<_{J}$ $h\left(x_{j}\right)$ " cannot occur more than $\mathrm{J}-\operatorname{depth}_{S}(h(x))$ times, so to bound $n$ we still have to bound the length of chains in Nulli of $S$ of the form $x_{m}<_{J} x_{m-1}<_{J} \cdots<_{J} x_{0}$ with $h\left(x_{m}\right) \equiv_{J} h\left(x_{m-1}\right) \equiv_{J} \cdots \equiv_{J} h\left(x_{0}\right)$ in $S$.

For each such $x_{k}<_{J} x_{k-1}$ with $h\left(x_{k}\right) \equiv_{J} h\left(x_{k-1}\right)$ we have $x_{k}=a_{k} x_{k-1} b_{k}$ for some $a_{k}, b_{k} \in X \cup\{1\}$, and we can write either:

(1) $x_{k}<_{L} x_{k-1}\left(\right.$ here $\left.b_{k}=1\right)$, or

(2) $x_{k}<_{R} x_{k-1}\left(\right.$ here $\left.a_{k}=1\right)$, or

(3) $x_{k} \leqslant R \quad u_{k}<_{L} x_{k-1}$ (where $u_{k}=a_{k} x_{k-1}$ ), or

(4) $x_{k} \leqslant L u_{k}<_{R} x_{k-1}$ (where $u_{k}=x_{k-1} b_{k}$ ). 
The cases $x_{k}<_{L} u_{k} \equiv_{R} x_{k-1}$ and $x_{k}<_{R} u_{k} \equiv_{L} x_{k-1}$ can be ignored; if they occur we simply replace $x_{k-1}$ by $u_{k}$ as representative of that $J$-class.

In any case we obtain the following $L$-chain and $R$-chain:

$$
\begin{gathered}
a_{m} a_{m-1} \ldots a_{1} \leqslant_{L} a_{m-1} \ldots a_{1} \leqslant_{L} \ldots \ldots \leqslant_{L} a_{1} \text { in } X \\
b_{1} \geqslant_{R} b_{1} b_{2} \geqslant_{R} \ldots \ldots \geqslant_{R} b_{1} \ldots b_{m-1} \geqslant_{R} b_{1} \ldots b_{m-1} b_{m} \text { in } X .
\end{gathered}
$$

Also $x_{k}=a_{k} \ldots a_{1} x_{0} b_{1} \ldots b_{k}$ for $k=1, \ldots, m$.

Claim 1.

$$
\begin{gathered}
\text { length of }\left(x_{m}<_{J} \ldots<_{J} x_{1}<_{J} x_{0}\right) \\
\leqslant 1+\text { length of } \operatorname{red}_{L}\left(a_{m} a_{m-1} \ldots a_{1} \leqslant L \ldots \ldots \leqslant_{L} a_{1}\right) \\
+ \text { length of } \operatorname{red}_{R}\left(b_{1} \geqslant_{R} \ldots \ldots \geqslant_{R} b_{1} \ldots b_{m-1} b_{m}\right)
\end{gathered}
$$

(where $\operatorname{red}_{L}$, respectively $\operatorname{red}_{R}$, denote then reductions as defined for the Rhodes expansions $\wedge_{L}(S)$ respectively $\left.\wedge_{R}(S)\right)$.

Proof of Claim 1: We show that if $x_{k}<_{L} x_{k-1}$ or $x_{k} \leqslant_{R} u_{k}<_{L} x_{k-1}$ then $a_{k} a_{k-1} \ldots a_{1}<_{L} a_{k-1} \ldots a_{1}$. Indeed, suppose $a_{k} a_{k-1} \ldots a_{1} \equiv_{L} a_{k-1} \ldots a_{1}$. Multiplying this $L$-equivalence on the right by $x_{0} b_{1} \ldots b_{k-1}$ yields $a_{k} a_{k-1} \ldots a_{1} x_{0} b_{1} \ldots b_{k-1} \equiv_{L}$ $a_{k-1} \ldots a_{1} x_{0} b_{1} \ldots b_{k-1}$. Therefore, if $b_{k}=1$ (so $x_{k}=u_{k}<_{L} x_{k-1}$ ) we get $x_{k} \equiv_{L} x_{k-1}$ (a contradiction), and otherwise (when $x_{k} \leqslant R u_{k}<_{L} x_{k-1}$ ) we get $u_{k} \equiv_{L} x_{k-1}$ (again a contradiction).

In a similiar way one proves that if $x_{k}<_{R} x_{k-1}$ or $x_{k} \leqslant_{L} u_{k}<_{R} x_{k-1}$ then $b_{1} \ldots b_{k-1}>_{R} b_{1} \ldots b_{k-1} b_{k}$.

The claim follows immediately from that.

CLAIM 2. If $h\left(x_{m}\right) \equiv_{J} \cdots \equiv_{J} h\left(x_{1}\right) \equiv_{J} h\left(x_{0}\right)$ belongs to Nulli of $S$ then $\left\{a_{k} \ldots a_{1} \mid k=1, \ldots, m\right\}$ and $\left\{b_{1} \ldots b_{k} \mid k=1, \ldots, m\right\}$ belong to higher layers of $X$ (that is $\operatorname{Reg} 1 \cup \cdots \cup \operatorname{Null}(i-1) \cup \operatorname{Reg} i$ of $X$, excluding Nulli itself).

Proof of Claim 2: We will use the fact that $S$ has the Falling Property. Suppose, by contradiction, that $a_{k} \ldots a_{1} \in \mathrm{Null}_{X} i$, hence (since $h$ is $\left.J^{*}\right) h\left(a_{k} \ldots a_{1}\right) \in$ $\mathrm{Null}_{S} i$. Also $h\left(x_{k}\right) \equiv_{J} h\left(x_{0} b_{1} \ldots b_{k}\right) \equiv_{J} h\left(x_{0}\right)$ belong to Null $s_{s}$. Now, since $h\left(a_{k} \ldots a_{1}\right)$ and $h\left(x_{0} b_{1} \ldots b_{k}\right)$ belong to $\mathrm{Null}_{S} i$ there is no regular element $J$-between them, hence (Falling Property of $S$, by Fact 3): $h\left(a_{k} \ldots a_{1}\right) \cdot h\left(x_{0} b_{1} \ldots b_{k}\right)<J$ $h\left(x_{0} b_{1} \ldots b_{k}\right) \equiv J h\left(x_{0}\right)$. But this means $h\left(x_{k}\right)<_{J} h\left(x_{0}\right)$, contradicting our assumption that $h\left(x_{k}\right) \equiv_{J} h\left(x_{0}\right)$ for $k=1, \ldots, m$.

Since (by Claim 2) $a_{m} \ldots a_{1}$ and $b_{1} \ldots b_{m}$ belong to $\operatorname{Reg} 1 \cup \ldots \cup \operatorname{Null}(i-1) \cup$ Regi of $X$ they have a well defined finite $\mathrm{J}$-depth in $X$ (by induction hypothesis), and, moreover $\mathrm{J}$-depth$X\left(a_{m} \ldots a_{1}\right)$ and $\mathrm{J}_{-} \operatorname{depth}_{X}\left(b_{1} \ldots b_{m}\right)$ are bounded by 
$f\left(\mathrm{~J}-\operatorname{depth}_{S}\left(h\left(a_{m} \ldots a_{1}\right)\right)\right)$ and $f\left(\mathrm{~J}_{-} \operatorname{depth}_{S}\left(h\left(b_{1} \ldots b_{m}\right)\right)\right)$ respectively. Since $f$ is increasing and since $x_{m} \leqslant_{J} a_{m} \ldots a_{1}$ and $x_{m} \leqslant{ }_{J} b_{1} \ldots b_{m}$ we have: J-depth ${ }_{X}\left(a_{m} \ldots a_{1}\right)$ and $J$-depth ${ }_{X}\left(b_{1} \ldots b_{m}\right)$ are bounded by $f\left(J-\operatorname{depth}_{S}\left(h\left(x_{m}\right)\right)\right)$.

Let us now again consider an arbitrary $<_{J}$-chain in $\mathrm{Null}_{X} i$ ascending from $x: x<_{J}$ $x_{n-1}<_{J} \cdots<_{J} x_{0}$. The number of places $x_{i+1}<_{J} x_{i}$ in the chain where $h\left(x_{i+1}\right)<_{J}$ $h\left(x_{i}\right)$ is bounded by $\mathbf{J}$-depth ${ }_{S}(h(x))$, as remarked earlier. Also we proved that every subchain of the form $x_{m}<_{J} \cdots<_{J} x_{i}$ with $h\left(x_{m}\right) \equiv_{J} \cdots \equiv_{J} h\left(x_{i}\right)$ in $S$ has length bounded by (in the notation of Claim 1) $1+J_{-} \operatorname{depth}_{X}\left(a_{m} \ldots a_{i}\right)+J-\operatorname{depth}_{X}\left(b_{i} \ldots b_{m}\right)$ which is in turn bounded (as observed above) by $1+f\left(\mathrm{~J}\right.$-depth ${ }_{S}\left(h\left(x_{m}\right)\right)$ ) $+f\left(\mathrm{~J}-\right.$ depth $\left._{S} h\left(x_{m}\right)\right)$. Since of course $x \leqslant_{J} x_{m}$ the length of the chain $x_{m}<_{J} \cdots<_{J} x_{i}$ is bounded by $1+2 \cdot f\left(\mathrm{~J}-\operatorname{depth}_{S}(h(x))\right)$, which depends only on $h(x)$. So, finally $n+1$ (=length of chain $x<_{J} x_{n-1}<_{J} \cdots<_{J} x_{0}$ in Nulli) is bounded by $\mathrm{J}_{\text {-depth }} h(x) \cdot\left(1+2 \cdot f\left(\mathrm{~J}\right.\right.$-depth $\left.\left.{ }_{S} h(x)\right)\right)$. This enables us then to extend $f$ to Nulli of $X$.

Finally, to complete the inductive proof, assume that $J$-depth $X$ is defined on Reg $1 \cup \ldots \cup \operatorname{Reg} i \cup$ Nulli, (and also that $f$ is defined up to Nulli of $X$ ), and let us show that $J$-depth $X$ is also defined on $\operatorname{Reg}(i+1)$ on $X$ (that is, extend $f$ to the next regular layer).

Again consider $x \in \operatorname{Reg}(i+1)$ of $X$ and a chain $x<_{J} x_{n-1}<_{J} \cdots<_{J} x_{0}<_{J}$ $y_{P}<_{J} \cdots<_{J} y_{1}$, where $x, x_{n-1}, \ldots, x_{0} \in \operatorname{Reg}_{X}(i+1)$ and $y_{P}, \ldots, y_{1} \in \operatorname{Reg} 1 \cup \cdots \cup$ Regi $i$ Nulli of $X$. By the same reasoning as before $p$ is bounded by $f\left(J-\operatorname{depth}_{S} h(x)\right)$. Bounding $n$ here is easy: it follows immediately from the fact that $h$ is $J^{*}$ and the fact that $x, x_{n-1}, \ldots, x_{0}$ are regular (so the chain $h(x)<_{J} h\left(x_{n-1}\right)<_{J} \cdots<_{J} h\left(x_{0}\right)$ in $S$ is also strict), hence $n \leqslant \mathrm{~J}$-depth $(h(x))$.

\section{Counterexamples.}

The following examples show that the assumptions on $S$ (namely that $S$ is stable and that it has a J-depth function) are not redundant.

Example of a semigroup $S$ which has a J-depth function but is not stable and for which $\wedge_{L}\left(S_{A}\right)$ does not have a J-depth function.

Let $S$ be an extension of a regular stable simple semigroup $D$ by a Baer-Levi semigroup $B$ (see [8, vol. 2] for the definition of Baer-Levi semigroups); let $B$ act as the identity on $D$ (that is, if $s \in B$ and $t \in D$ then $s t=t s=t$ ). Then clearly $S$ is not stable but has a J-depth function.

Then $\wedge_{L}\left(S_{A}\right)$ will be an extension of a regular stable simple semigroup $D^{\prime}$ by the free semigroup $\wedge_{L} B_{A \cap B}$, since Rhodes proved [11] that $\wedge_{L} B_{A \cap B}$ is free. (Here $A$ is any set of generators of $S$, and $A \cap B$ is a set of generators of $B$.) Recall that in the map $\wedge_{L}\left(S_{A}\right) \rightarrow \rightarrow S$ the inverse image of a regular $J$-class $D$ is one regular $J$-class $D^{\prime}$, and the null-regular layer is preserved. Clearly now the elements of $D^{\prime} \subseteq \wedge_{L}\left(S_{A}\right)$ 
have infinite $\mathrm{J}$-depth .

The same reasoning works for $\wedge_{+}\left(S_{A}\right)$.

Example of a semigroup $S$ which is stable but does not have a J-depth function, and for which $\wedge_{L}\left(S_{A}\right), \wedge_{R}\left(S_{A}\right)$ and $\wedge_{+}\left(S_{A}\right)$ do not have a J-depth function.

Let $S$ be the extension of a regular stable simple semigroup $D$ by a free semigroup $F$, with identity action. Clearly $S$ is stable but elements in $D$ have infinite J-depth .

Then $\wedge_{L}\left(S_{A}\right)$ is again the extension of a regular simple semigroup $D^{\prime}$ by a free semigroup $F$ (since in the map $\wedge_{L}\left(S_{A}\right) \rightarrow \rightarrow S$ the inverse image of a regular $J$-class is one regular $J$-class). But again, in $\wedge_{L}\left(S_{A}\right)$ the elements of $D^{\prime}$ have infinite J-depth . The same reasoning works for $\wedge_{R}\left(S_{A}\right)$ and $\wedge_{+}\left(S_{A}\right)$.

\section{Refenences}

[1] J.C. Birget, 'Iteration of expansions - Unambiguous semigroups', J. Pure Appl. Algebra 34 (1984), 1-55.

[2] J.C. Birget, 'Arbitrary vs. regular semigroups', J. Pure Appl. Algebra 34 (1984), 56-115.

[3] J.C. Birget, 'Structure of finite semigroups, and generalisations', (K. Byleen, P. Jones, F. Pastijn editors), Proceedings of the 1984 Marquette Conference on Semigroups, 1-15.

[4] J.C. Birget, 'The Synthesis Theorem for finite regular semigroups and its generalisation' (to appear).

[5] J.C. Birget, S. Margolis and J. Rhodes, 'Finite semigroups whose idempotents from a semilat tice or a band' (unpublished), see also, Proceedings of the Chico Conference on Semigroups.

[6] J.C. Birget and J. Rhodes, 'Almost finite expansions of arbitrary semigroups', J. Pure Appl. Algebra 32 (1984), 239-287.

[7] T.C. Brown, 'An interesting combinatorial method in the theory of locally finite semigroups', Pacific J. Math. 36 (1971), 285-289.

[8] A.H. Clifford and G. Preston, The Algebraic Theory of Semigroups, Vol. 1 (1961), Vol. 2 (1967). (Math. Surveys Monogr., American Mathematical Society, Providence, R.I.) .

[8] S. Eilenberg, Automata, Languages, and Machines (Academic Press, New York, 1976).

[10] G. Lallement, Semigroups and Combinatorial Applications (John Wiley, New York, 1979).

[11] J. Rhodes, 'Infinite iteration of matrix semigroups, Part II', J. Algebra 100 (1986), 25-137.

[12] J. Rhodes, Algebraic and topological theory of languages and computation, (Preprint (1983), Center for Pure Applied Math., U. of California, Berkeley).

[13] J. Rhodes, 'Expansions of semigroups via Ramsey's and Brown's theorem', (in progress).

[14] J. Rhodes and D. Allen, 'Synthesis of classical and modern semigroup theory', Adv. in Math. 11 (1976), 238-266.

[15] J. Rhodes and B. Tilson; Machines, Languages and Semigroups, Chapters 7 and 8 (M. Arbib, editor, Academic Press, New York, 1968).

[16] H. Straubing, 'The Burnside problem for semigroups of matrices', in Combinatorics on Works (L. Cummings editor, Academic Press, New York, 1983).

[17] B. Tilson, 'On the complexity of finite semigroups', J. Pure Appl. Algebra 4 (1974).

Department of Computer Science

University of Nebraska

Lincoln, NE 68588-0115

United States of America. 